BMJ Open

Diabetes

Research

\& Care

\section{Bacterial infections in patients with type 1 diabetes: a 14-year follow-up study}

To cite: Simonsen JR, Harjutsalo V, Järvinen A, et al. Bacterial infections in patients with type 1 diabetes: a 14-year follow-up study. BMJ Open Diabetes Research and Care 2015;3:e000067. doi:10.1136/bmjdrc-2014000067

- Additional material is available. To view please visit the journal (http://dx.doi.org/ 10.1136/bmjdrc-2014000067).

$\overline{\text { JRS and VH }}$ contributed equally.

Received 7 November 2014 Revised 9 January 2015 Accepted 15 February 2015

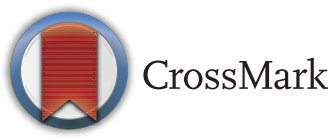

For numbered affiliations see end of article.

Correspondence to Dr Markku Lehto; markku. lehto@helsinki.fi

\begin{abstract}
Johan R Simonsen, ${ }^{1,2,3}$ Valma Harjutsalo, ${ }^{1,2,3,4}$ Asko Järvinen, ${ }^{5}$ Juha Kirveskari, ${ }^{6}$ Carol Forsblom, ${ }^{1,2,3}$ Per-Henrik Groop, ${ }^{1,2,3,7}$ Markku Lehto, ${ }^{1,2,3}$ on behalf of the FinnDiane Study Group
\end{abstract}

\section{ABSTRACT}

Objective: This study explored the annual occurrence/ incidence of bacterial infections, and their association with chronic hyperglycemia and diabetic nephropathy, in patients with type 1 diabetes.

Design: In a register-based follow-up study, we investigated the frequency of bacterial infections in patients with type 1 diabetes $(n=4748)$ and agematched and sex-matched non-diabetic control (NDC) subjects ( $n=12$ 954) using nationwide register data on antibiotic drug prescription purchases and hospital discharge diagnoses, collected between 1996 and 2009. Diabetic nephropathy was classified based on the urinary albumin excretion rate (AER).

Results: The hospitalization rate due to bacterial infections was higher in patients with diabetes compared with NDCs (rate ratio (RR) $2.30(95 \% \mathrm{Cl}$ 2.11 to 2.51)). The rate correlated with the severity of diabetic nephropathy: RR for microalbuminuria was 1.23 (0.94 to 1.60), 1.97 (1.49 to 2.61) for macroalbuminuria, 11.2 (8.1 to 15.5) for dialysis, and 6.72 (4.92 to 9.18) for kidney transplant as compared to patients with diabetes and normal AER. The annual number of antibiotic purchases was higher in patients with diabetes (1.00 (1.00 to 1.01)) as compared with NDCs (0.47 (0.46 to 0.47$)), \mathrm{RR}=1.71$ (1.65 to 1.77 ). Annual antibiotic purchases were 1.18-fold more frequent in patients with microalbuminuria, 1.29-fold with macroalbuminuria, 2.43-fold with dialysis, and 2.74-fold with kidney transplant as compared to patients with normal AER. Each unit of increase in glycated hemoglobin was associated with a $6-10 \%$ increase in the number of annual antibiotic purchases.

Conclusions: The incidence of bacterial infections was significantly higher in patients with type 1 diabetes compared with age-matched and sex-matched NDC subjects, and correlated with the severity of diabetic nephropathy in inpatient and outpatient settings.

\section{INTRODUCTION}

Diabetes has previously been associated with an increased risk of infections and mortality due to infectious diseases. ${ }^{1-3}$ Patients with diabetes have a twofold higher risk of community-acquired enterobacterial, pneumococcal, and streptococcal bacteremia as

\section{Key messages}

- The prevalence of bacterial infections is greater in patients with diabetes compared with nondiabetic control subjects, and correlates with the severity of diabetic nephropathy.

- Poor glycemic control is associated with an increased number of antibiotic purchases in patients with diabetes.

- Frequent use of antibiotics is associated with an increased risk of the development of nephropathy in patients with diabetes.

compared with patients without diabetes. ${ }^{4-6}$ Type 1 diabetes in particular has been associated with various defects in the innate and the adaptive immune system, which in turn may increase the risk of infections. ${ }^{7-9}$ Poor glycemic control has been shown to be an important risk factor for infections. ${ }^{1}{ }^{6} 10$ Even acute hyperglycemia has been associated with elevated levels of inflammation markers in patients with diabetes. ${ }^{11}{ }^{12}$ Notably, a $1 \mathrm{mmol} / \mathrm{L}$ increase in plasma glucose has been associated with a $6-10 \%$ increased risk of hospitalization for pneumonia, urinary tract infections, and skin infections. ${ }^{13}$

Apart from hyperglycemia, other complications of diabetes may also predispose patients to infections. Diabetic neuropathy can cause impaired bladder emptying and thereby increase the susceptibility to urinary tract infections. $^{14}$ Neuropathy in combination with peripheral vascular disease can lead to ulcerations in the skin and secondary infections. ${ }^{15}$ Long duration of diabetes increases the risk of microvascular and macrovascular complications and it has been estimated that during their lifetime, approximately onethird of patients with type 1 diabetes will develop diabetic nephropathy, which is the most common cause of chronic kidney disease and end-stage renal disease (ESRD). Owing to dialysis treatment and the lifelong immunosuppressive drug therapy that follows 
kidney transplantation, ESRD has been associated with an increased risk for bacterial infections. ${ }^{16}$ However, studies have also shown that patients with chronic kidney disease have an increased risk of infections and infection-related mortality already before ESRD, caused by reduced glomerular filtration rate (GFR) and higher urinary albumin excretion rate (AER). ${ }^{17}{ }^{18}$ Chronic inflammation, in turn, has been associated with the progression of diabetic nephropathy. ${ }^{19} 20$

Earlier studies have shown an increased risk of bacterial infections in patients with diabetes; however, most of these studies have focused on specific pathogens or specific infection sites such as skin, urinary tract, and respiratory infections or severe infections necessitating hospitalization. ${ }^{4-6} 1013$ Consequently, the overall risk of infectious diseases in patients with diabetes, both severe infections and less severe infections treated outside of hospitals, has not been established. Further, many of the infection-related studies have not separated patients with type 1 and type 2 diabetes. The principal aim of the present study was therefore to assess the overall risk of bacterial infections in patients with type 1 diabetes in inpatient and outpatient settings. As a secondary aim, we wanted to study the association with bacterial infections and poor glycemic control, as well as their possible association with diabetic nephropathy.

\section{METHODS}

\section{Design overview}

Patients with type 1 diabetes were recruited, characterized, and prospectively followed by the Finnish Diabetic Nephropathy (FinnDiane) Study. The FinnDiane Study is an ongoing nationwide multicenter survey, founded in 1997 to elucidate genetic and environmental risk factors for diabetic nephropathy in patients with diabetes. The study protocol is in accordance with the declaration of Helsinki, and it has been approved by the local ethics committee at each study center. During the baseline visit, details on clinical status, including age at diagnosis, insulin therapy, and other medications, as well as presence and severity of diabetic complications are registered by a standardized questionnaire, which is completed by the patient's attending physician based on medical files. Fasting blood samples were collected during the baseline visit from the patients for the measurement of glycated hemoglobin (HbAlc), which was determined by standardized assays at each study center. After the baseline visit, the patients were followed and re-examined with prospective visits approximately in 3-5-year intervals. Subsequent urine analyses were conducted for the follow-up of urinary AER for the possible detection of the development and progression of diabetic nephropathy. Nephropathy data were also collected from the medical records.

\section{Setting and participants}

Follow-up started in 1996 and ended at death or at the end of 2009. The FinnDiane patient cohort constituted of 4748 patients with type 1 diabetes, of whom $52.7 \%$ were males. For each patient with diabetes, three nondiabetic control (NCD) subjects who were matched for sex, age, and place of residence in the year of diagnosis of diabetes were selected from the Finnish Public Register Centre. After exclusion of individuals who died or moved abroad before 1996, and those who were entitled to special reimbursement of drug cost for diabetes mellitus between 1996 and 2009, the final number of control subjects was 12954 (51.6\% males). Type 1 diabetes was defined as age at onset $<40$ years and permanent insulin treatment started within 1 year after the diagnosis of diabetes.

The severity of diabetic nephropathy was assessed by the AER in at least two of three overnight or $24 \mathrm{~h}$ urine collections: normal AER $(<20 \mu \mathrm{g} / \mathrm{min}$ or $<30 \mathrm{mg} / 24 \mathrm{~h})$, microalbuminuria $(\geq 20<200 \mu \mathrm{g} / \mathrm{min}$ or $\geq 30<300 \mathrm{mg}$ / $24 \mathrm{~h})$, macroalbuminuria $(\geq 200 \mu \mathrm{g} / \mathrm{min}$ or $\geq 300 \mu \mathrm{g} /$ $24 \mathrm{~h}$ ), and ESRD (defined as dialysis treatment or kidney transplantation). Urinary samples were not collected, if the patients had fever or female patients were menstruating. The time point for detection of nephropathy progression was defined as the year when the AER, in subsequent urine samples collected during scheduled prospective visits or measured in conjunction with hospital visits, had in at least two of three samples increased to the following stage of nephropathy, that is, microalbuminuria or macroalbuminuria, or for ESRD the date of the first dialysis or kidney transplant. The AER was not available at baseline for 579 patients. The final number of patients with confirmed AER was therefore 4169. Altogether, there were 2664 patients with normal AER, 544 with microalbuminuria, 625 with macroalbuminuria, 85 with dialysis, and 251 with kidney transplantation at the baseline visit. During the follow-up time, $8.2 \%$ of the patients with normoalbuminuria, $18.2 \%$ with microalbuminuria, and $39.4 \%$ with macroalbuminuria progressed to a higher level of albuminuria or ESRD. Of the patients on dialysis, $27.1 \%$ underwent kidney transplantation during the follow-up. Estimated glomercular filtration rates (eGFR), calculated with the Chronic Kidney Disease Epidemiology Collaboration (CKD-EPI) formula, were used in the analyses as a complementary method for diabetic kidney disease progression. When the analyses were conducted by eGFR, those patients with unknown AER could also be included. Instead, the patients with ESRD were excluded.

\section{Data sources and outcomes}

Information on hospitalizations due to bacterial infections and on antimicrobial drug prescription purchases for each participant was drawn from two nationwide registries: the Finnish Hospital Discharge Register, which includes the diagnoses of all in-hospital treatment periods, and the Finnish National Drug Prescription Register, which includes all of the antibiotics purchased from pharmacies outside hospitals. Infections treated in hospitals were classified according to their most 
common etiology as bacterial, viral, fungal, or as unspecified infections, using the primary and secondary diagnoses from the Hospital Discharge Register, according to the WHO International Classification of Diseases (ICD) 10th revision. The specific types and sites of the hospital-treated infections are more closely specified in online supplementary table S1. The risk of infections in outpatients was assessed by acquiring information on the annual outpatient prescription purchase frequencies of antibiotics. Antibiotics were identified using the WHO Anatomical Therapeutic Chemical (ATC) Classification System as drugs with an ATC code beginning with J01.

Since comorbidities may have an effect on the risk of infections, common comorbidities were identified from the Hospital Discharge Register, the Drug Prescription Register, and the Drug Reimbursement Register for patients with diabetes and NDCs. These comorbidities included hypertension, cardiovascular disease (CVD including ischemic heart disease and stroke), atherosclerosis, cancers, mental disorders, neurological diseases, alcoholism, autoimmune diseases such as rheumatoid arthritis, and respiratory disease (asthma and obstructive pulmonary disease) as they belong to the most common diseases in adults. The codes indicating certain diseases are available in online supplementary table S2.

In a subanalysis, the number of antibiotic purchases was compared between patients who had retained a normal AER during the whole follow-up period $(n=2445)$ with their respective sex-matched and agematched NDCs $(n=6560)$ to separate between the impact of nephropathy and other possible effects of diabetes on antibiotic purchases.

In order to study more closely the association between glycemic control and bacterial infections in patients with diabetes, we examined the impact of the baseline HbAlc on the total number of antibiotic purchases made within a 3-year period: 1 year before the baseline visit, during the examination year, and 1 year after the baseline visit. The baseline HbA1c values were categorized into five groups: $<7 \%, 7-7.9 \%, 8-8.9 \%, 9-9.9 \%$, and $\geq 10 \%$. Results are presented as incidence rate ratios (RRs) with 95\% CIs with the lowest value as reference. The analyses were conducted separately in patients with normal AER, and in patients with microalbuminuria or macroalbuminuria. Patients with unknown nephropathy status were excluded from these analyses.

Finally, the potential association between bacterial infections and the development of diabetic nephropathy was studied in a subset of patients with diabetes who developed microalbuminuria during our follow-up period $(n=219)$. The annual number of antibiotic purchases was counted from up to 4 years before, and 3 years after, the year of the diagnosis of incident microalbuminuria. For each of the patients, we assigned sex, age, and diabetes duration ( \pm 2 years) matched controls with diabetes who had retained a normal AER $(n=874)$ during the whole follow-up period. For these controls, the number of antibiotic purchases was counted for the same years as their corresponding patients with incident microalbuminuria.

\section{Statistical analysis}

The cumulative number of hospitalizations due to infections and the number of antibiotic purchases were calculated for each individual annually from 1996 until death or the end of the year 2009. In case of no hospitalization or antibiotic purchase, the outcome was set to zero. When the analyses were conducted according to nephropathy groups, the follow-up started from the baseline visit. The patients contributed the data during each year to the corresponding nephropathy group. Since there were only a few baseline visits in the years 1996 and 1997 in each nephropathy group, these results are shown between 1998 and 2009.

The count data were highly skewed with excess zeros. Thus, zero-inflated Poisson (ZIP) models were fitted to the data using the SAS NLMIXED procedure, ${ }^{21}$ which takes into account the excess of zeros. Since one person may have had multiple events, subject-specific random effects were incorporated into the models. When the outcomes between the patients with and without diabetes were compared, the predictors were calendar year of the event and comorbidities. When the analyses were conducted separately for these groups, the age in the year 1996 and sex were included as covariates. In the analyses according to nephropathy groups, the covariates were age and duration of diabetes at baseline visit, sex, calendar year, HbA1c, eGFR (not in the patients with ESRD), smoking status, and comorbidities. The RRs of hospitalization rates or antibiotic purchases and the $95 \%$ CIs as well as predicted values were derived from the final ZIP models. Statistical analysis was performed using the Statistical Analysis System (SAS) software (SAS 9.3, Cary, North Carolina, USA).

\section{RESULTS}

The study cohort comprised of 12954 NDCs and 4748 patients with type 1 diabetes (table 1). Between 1996 and 2009, the total number of follow-up years was 177268 in the NDC group and 64194 in patients with type 1 diabetes. The total number of follow-up years in each nephropathy group was as follows: normal AER (19 804 years), microalbuminuria (4958 years), macroalbuminuria (5821 years), dialysis (856 years), and kidney transplant (2250 years). As expected, the median duration of diabetes increased along with increasing severity of nephropathy. Hypertension, asthma, and mental disorders were the most common comorbidities in patients with diabetes and in NDCs. CVD and atherosclerosis were much more frequent in patients with diabetes (17.2\% and $8.1 \%)$ compared with NDCs $(3.9 \%$ and $0.3 \%)$ as they are well-known macrovascular complications of diabetes. Over $10 \%$ of patients with diabetes died during follow-up as compared to $4.4 \%$ of NDCs. 


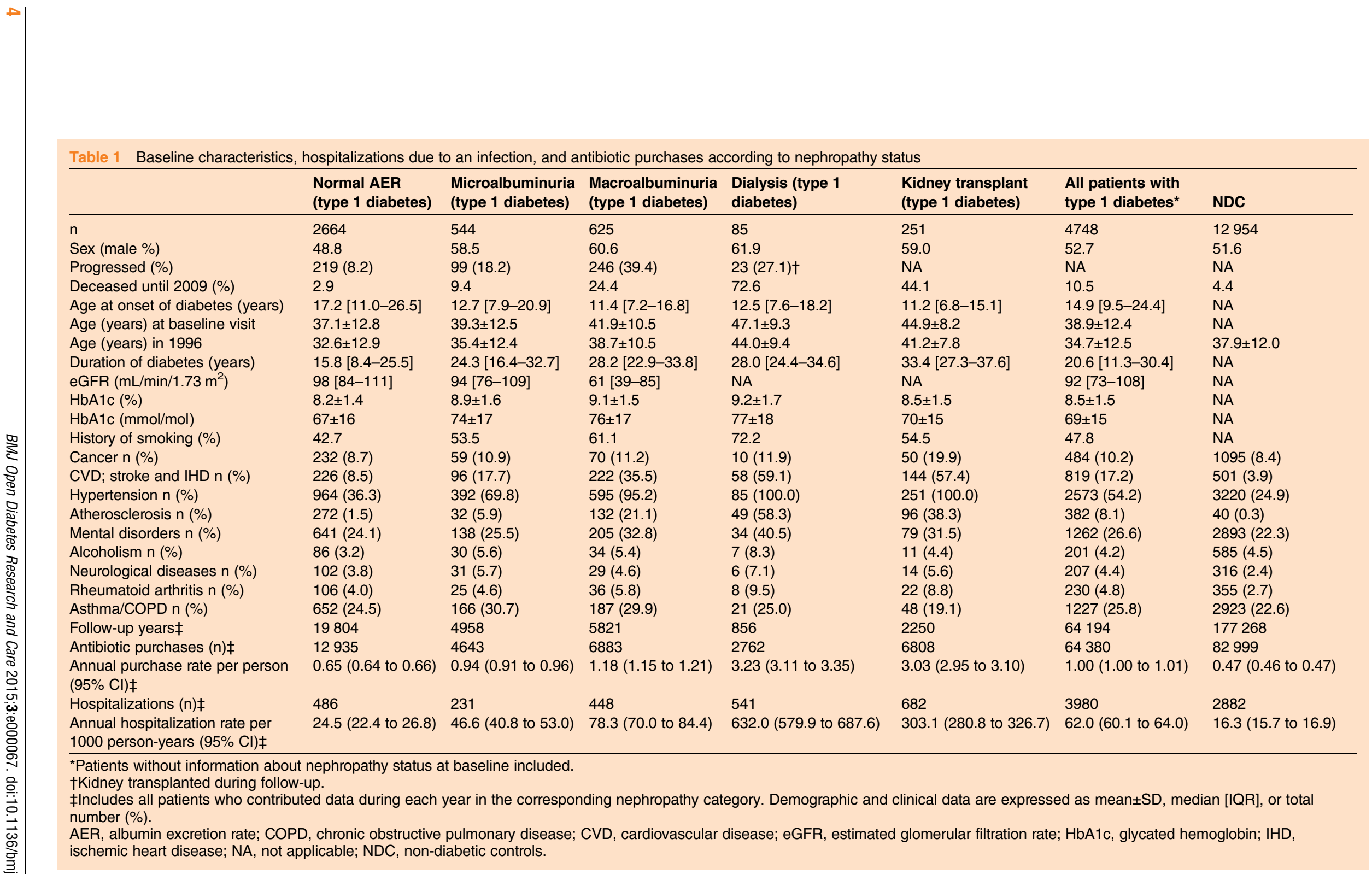




\section{Hospitalization due to infections}

Data on infections leading to hospitalization were obtained from the Finnish Hospital Discharge Register (table 1). The patients with diabetes had a total of 3980 hospitalization events due to infections, of which 3229 $(81.1 \%)$ were due to bacterial infection. The corresponding number of events in the NDCs was 2882, where $2102(72.9 \%)$ infections had a bacterial etiology (online supplementary table S1). The hospitalization rate was higher in patients with diabetes compared with NDCs, RR=2.30 (95\% CI 2.11 to 2.51) in an adjusted model. Patients with diabetes displayed an increasing trend of hospitalizations between 1996 and 2009 with a $4 \%$ annual increase. In contrast, NDCs showed a slightly decreasing trend with a $3 \%$ annual reduction in hospitalizations (table 2). Increasing age had a decreasing effect on hospitalization risk in patients with diabetes and NDCs. Men and women were equally hospitalized in the diabetes group. In contrast, women in the NDC group had a $44 \%$ lower hospitalization rate $(R R=0.56$, $95 \%$ CI 0.50 to 0.62 , table 2) compared with men. In both groups, all comorbidities increased the risk of hospitalization except rheumatoid arthritis in the diabetes group. Among comorbidities, atherosclerosis had the largest effect in both groups and it increased the risk of hospitalizations threefold: $\mathrm{RR}=3.16 \quad(95 \%$ CI 3.11 to 5.45) for patients with diabetes; $\mathrm{RR}=3.09$ (95\% CI 1.87 to 3.29) for NDCs (table 2).

Time trends for annual hospitalization rates in different nephropathy classes are shown in online supplementary figure S1. The hospitalization rate increased with the severity of diabetic nephropathy: the mean hospitalization rate per 1000 person-years in patients with normal AER was 24.5 (95\% CI 22.4 to 26.8), in patients with microalbuminuria 46.6 (95\% CI 40.8 to 53.0), and in those with macroalbuminuria 78.3 (95\% CI 70.0 to 84.4 ; table 1 ). In patients with ESRD, those on dialysis had a higher hospitalization rate compared with patients with a kidney transplant; 632 (95\% CI 580 to 688 ) versus 303 (95\% CI 281 to 327) per 1000 personyears. The RRs when compared with the normoalbuminuria group were 1.23 (95\% CI 0.94 to 1.60$), 1.97$ (95\% CI 1.49 to 2.61), 11.2 (95\% CI 8.1 to 15.5 ), and 6.72 (95\% CI 4.92 to 9.18) for the microalbuminuria, macroalbuminuria, dialysis, and kidney transplantation groups, respectively. Hospitalizations were also inversely related to eGFR (online supplementary figure S3). The risk was the lowest and rather stable when eGFR was higher than $80 \mathrm{~mL} / \mathrm{min} / 1.73 \mathrm{~m}^{2}$, but started to increase rapidly when eGFR showed reduced kidney function.

Online supplementary table S3 shows the hospitalization RRs obtained from multivariate ZIP modeling according to nephropathy status. Hyperglycemia was strongly associated with hospitalizations in all other groups except those on dialysis. Even in patients with normal AER, each unit increase in HbAlc increased hospitalizations by $26 \%$.

\section{Outpatient purchases of antibiotics}

Data on antibiotic purchases were obtained from the Finnish National Drug Prescription Register (table 1). Patients with diabetes purchased more antibiotics annually compared with NDCs; 1.00 (95\% CI 1.00 to 1.01 ) vs $0.47(95 \%$ CI 0.46 to 0.47$) \quad(\mathrm{RR}=1.71 \quad(95 \%$ CI 1.65 to 1.77)). Antibiotic purchases increased during the study period in patients with diabetes (2\% annually) and in NDCs (1\% annually; table 2). Similarly, as for hospitalizations, increasing age had a decreasing effect on antibiotic purchases in patients with diabetes and NDCs. In both groups, female patients purchased around 50\% more antibiotics; RR=1.54 (95\% CI 1.46 to 1.63$)$ for patients with diabetes, 1.49 (95\% CI 1.44 to 1.55 ) for NDCs. Atherosclerosis had the most conspicuous comorbidity effect in patients with diabetes $(\mathrm{RR}=2.94$

Table 2 Multivariate ZIP-modeling and incidence RRs $(95 \% \mathrm{Cl})$ of the hospitalization due to infections and antibiotic purchases in all patients with type 1 diabetes and NDCs

\begin{tabular}{|c|c|c|c|c|}
\hline & \multicolumn{2}{|l|}{ Hospitalizations } & \multicolumn{2}{|c|}{ Antibiotic purchases } \\
\hline & $\begin{array}{l}\text { All patients with } \\
\text { type } 1 \text { diabetes }\end{array}$ & NDC & $\begin{array}{l}\text { All patients with } \\
\text { type } 1 \text { diabetes }\end{array}$ & NDC \\
\hline Age (years) & 0.98 (0.98 to 0.99$)$ & 0.97 (0.97 to 0.98$)$ & 0.99 (0.99 to 0.99$)$ & 0.99 (0.99 to 0.99$)$ \\
\hline Sex (men as reference) & 0.95 (0.84 to 1.07$)$ & 0.56 (0.50 to 0.62$)$ & 1.54 (1.46 to 1.63$)$ & $1.49(1.44$ to 1.55$)$ \\
\hline Calendar year & $1.04(1.03$ to 1.05$)$ & 0.97 (0.96 to 0.98$)$ & $1.02(1.02$ to 1.02$)$ & $1.01(1.01$ to 1.01$)$ \\
\hline Hypertension & 2.45 (2.12 to 2.83$)$ & 1.59 (1.40 to 1.82$)$ & 1.56 (1.47 to 1.66$)$ & $1.31(1.26$ to 1.37$)$ \\
\hline CVD hard event & 2.13 (1.81 to 2.49$)$ & 1.94 (1.53 to 2.47$)$ & 1.33 (1.23 to 1.44$)$ & 0.97 (0.88 to 1.06$)$ \\
\hline Atherosclerosis & $3.16(3.11$ to 5.45$)$ & 3.09 (1.87 to 3.29$)$ & 2.94 (2.66 to 3.25$)$ & 1.76 (1.29 to 2.38$)$ \\
\hline Cancer & 1.23 (1.02 to 1.48$)$ & 2.50 (2.10 to 2.96$)$ & $1.14(1.04$ to 1.24$)$ & $1.24(1.17$ to 1.33$)$ \\
\hline Mental disorder & $1.42(1.25$ to 1.62$)$ & $1.28(1.13$ to 1.45$)$ & 1.24 (1.17 to 1.32$)$ & $1.31(1.26$ to 1.37$)$ \\
\hline Alcoholism & 2.02 (1.57 to 2.59$)$ & 1.76 (1.41 to 2.18$)$ & 1.16 (1.02 to 1.32$)$ & $1.01(0.93$ to 1.10$)$ \\
\hline Neurological disease & 1.67 (1.30 to 2.15$)$ & 2.16 (1.64 to 2.85$)$ & 1.13 (0.99 to 1.28$)$ & $1.10(0.99$ to 1.24$)$ \\
\hline Rheumatoid arthritis & 1.24 (0.97 to 2.15$)$ & 1.61 (1.21 to 2.14$)$ & 1.15 (1.02 to 1.29$)$ & $1.36(1.23$ to 1.51$)$ \\
\hline Respiratory diseases (asthma/COPD) & 1.18 (1.03 to 1.34$)$ & $1.62(1.44$ to 1.82$)$ & $1.47(1.38$ to 1.56$)$ & $1.97(1.89$ to 2.05$)$ \\
\hline
\end{tabular}


(95\% CI 2.66 to 3.25$)$ ), while respiratory diseases did the same in the NDCs $(\mathrm{RR}=1.97$ (95\% CI 1.89 to 2.05$)$; table 2).

Patients with diabetes, who had retained a normal AER during the whole follow-up period, had more antibiotic purchases than NDCs, RR=1.56 (95\% CI 1.49 to 1.64). The risk ratio was only slightly decreased when further controlled for comorbidities ( $R R=1.48$ (95\% CI 1.41 to 1.55$)$ ). Time trends for annual antibiotic purchases in different nephropathy classes are shown in online supplementary figure 2. The annual number of purchases per person was 0.65 (95\% CI 0.64 to 0.66 ) in patients with normal AER, 0.94 (95\% CI 0.91 to 0.96 ) in patients with microalbuminuria, 1.18 (95\% CI 1.15 to 1.21 ) in patients with macroalbuminuria, 3.23 (95\% CI 3.11 to 3.35 ) in patients on dialysis, and 3.03 (95\% CI 2.95 to 3.10 ) in patients with a kidney transplant (table 1). The RRs obtained from the fully adjusted model were 1.18 (95\% CI 1.07 to 1.30 ) for microalbuminuria, 1.29 (95\% CI 1.15 to 1.44 ) for macroalbuminuria, $2.43(95 \%$ CI 2.08 to 2.84) for dialysis, and 2.74 (95\% CI 2.35 to 3.17) for the kidney transplantation group compared to patients with normal AER. Similarly, as for the hospitalizations, a reduced eGFR was associated with increased antibiotic purchases (online supplementary figure 3). There was a modest increase in purchases with decreasing eGFR until $90 \mathrm{~mL} / \mathrm{min} / 1.73 \mathrm{~m}^{2}$, and this increase was accelerated when eGFR decreased below $90 \mathrm{~mL} /$ $\min / 1.73 \mathrm{~m}^{2}$.

Online supplementary table S3 shows the antibiotic purchase RRs obtained from multivariate ZIP modeling according to nephropathy status. High HbAlc increased purchases with a range of $6-10 \%$ per unit increase in HbA1c across the nephropathy groups except in the patients with ESRD. The impact of glycemic control on antibiotic purchases was studied separately in (1) patients with normal AER and (2) patients with microalbuminuria or macroalbuminuria. Among patients with microalbuminuria and macroalbuminuria, patients with poor glycemic control (HbA1c $\geq 9 \%$ ) had 1.25 (95\% CI 1.08 to 1.45) times more antibiotic purchases than those with good glycemic control $(\mathrm{HbA} 1 \mathrm{c}<7 \%)$. In contrast, the number of antibiotic purchases in patients with normal AER was higher already when $\mathrm{HbAlc}$ was $\geq 7 \% \quad(\mathrm{RR}=1.22$ (95\% CI 1.14 to 1.31$)$ ). In patients with normal AER, the number of antibiotic purchases leveled off when HbAlc was $\geq 8 \%$ and in patients with microalbuminuria or macroalbuminuria when $\mathrm{HbAlc}$ was $\geq 9 \%$ (figure 1 ).

We also wanted to assess whether the frequent use of antibiotics could be associated with the progression of nephropathy. As seen in figure 2, the mean number of antibiotic purchases was higher in the group that developed microalbuminuria compared with the reference group with normal AER. In patients with incident microalbuminuria, the number of antibiotic purchases was higher at the time of nephropathy diagnosis (year 0 ) compared with 4 years before the progression (year -4 ; $\mathrm{p}=0.04$ for time effect). In contrast, no significant differences were observed in the number of antibiotic purchases in patients with normal AER during the follow-up $(\mathrm{p}=0.52$ for time effect).

\section{DISCUSSION}

We performed a survey on bacterial infections in one of the largest cohorts of patients with type 1 diabetes in the world. By combining data from two different nationwide registries that are mandatorily used in Finland, we were able to study the incidence of infections causing hospitalization as well as infections treated in the outpatient setting. Owing to the large number of patients and follow-up years, we were also able to study the association between bacterial infections and diabetic nephropathy. We observed that bacterial infections were more
Figure 1 Association between antibiotic purchases and glycated hemoglobin $(\mathrm{HbA} 1 \mathrm{c})$ values in patients with and without diabetic nephropathy. Patients with normal albumin excretion rate and microalbuminuria/ macroalbuminuria are shown as open and closed squares, respectively. $p$ Values $\left({ }^{\dagger} p<0.05\right.$; $\left.{ }^{*} p<0.001\right)$.

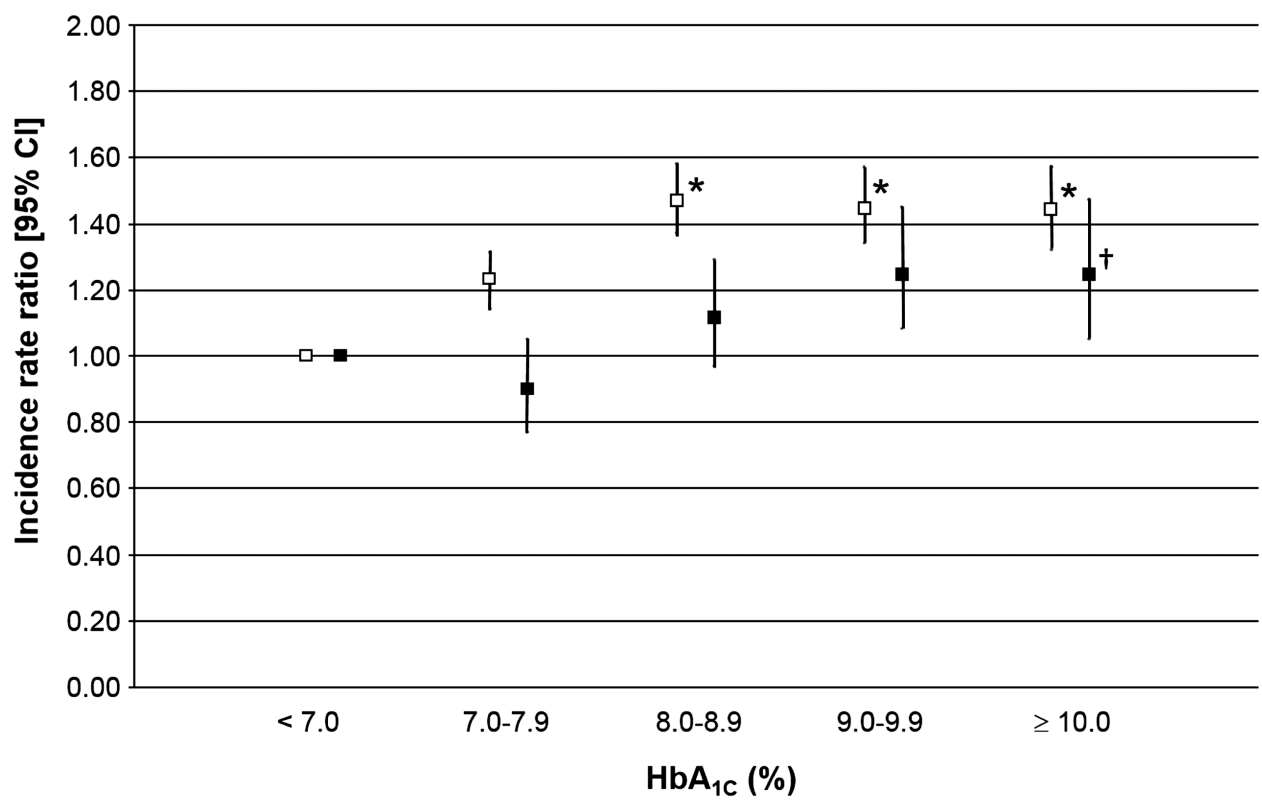




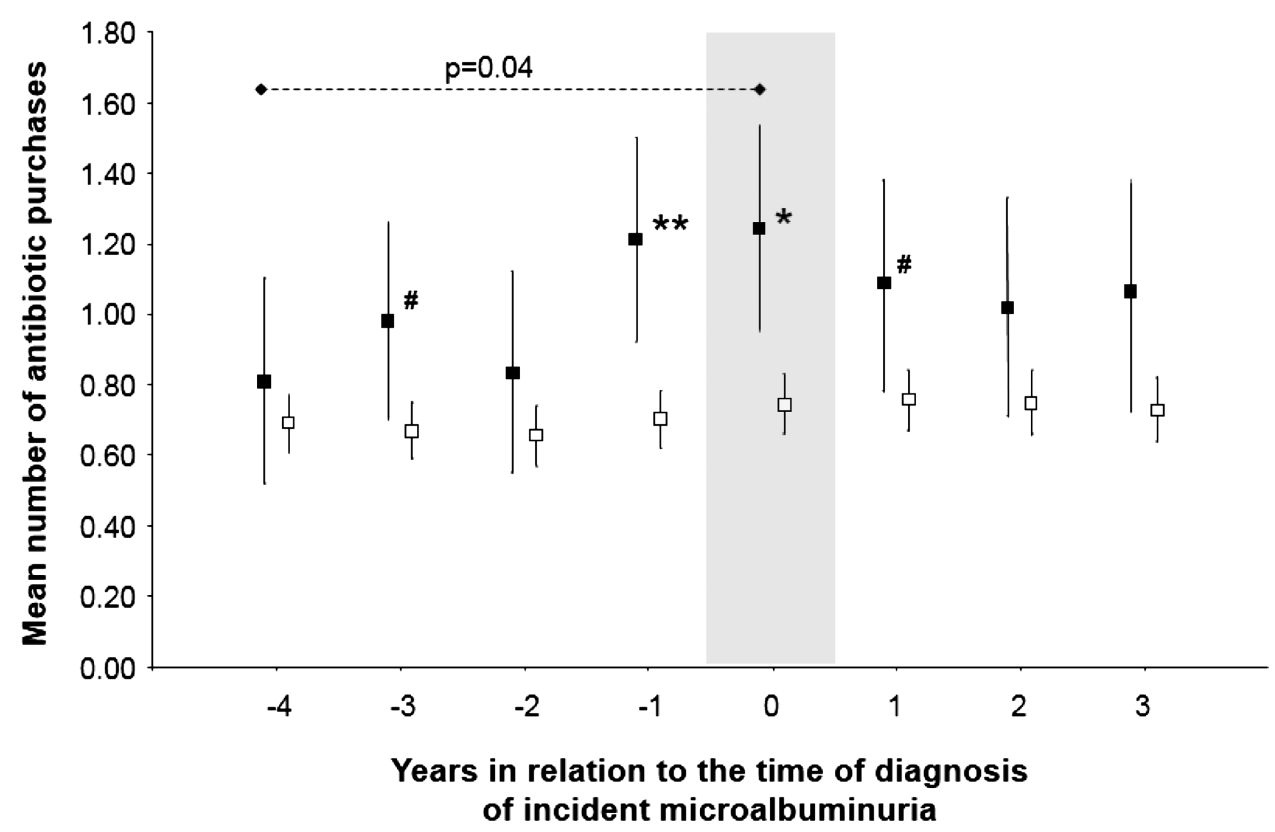

Figure 2 Relationships between annual antibiotic purchase rates and progression of microalbuminuria in patients with type 1 diabetes. Annual number of antibiotic purchases in patients with type 1 diabetes and incident microalbuminuria (progressors, $n=219$; closed squares), and their age, sex, and diabetes duration ( \pm 2 years) matched controls with type 1 diabetes that have retained a normal albumin excretion rate during the whole follow-up period (non-progressors, $n=874$; open squares). The first year of incident microalbuminuria is shown as year 0 . Controls were matched for sex, age, and diabetes duration ( \pm 2 years). Values are expressed as means with $95 \% \mathrm{Cls}$, and were adjusted for sex, $\mathrm{HbA1c}$, and diabetes duration. A dashed horizontal line indicates the level of significance between years -4 and 0 in the incident microalbuminuria group. $p$ Values $\left({ }^{\#} p<0.05\right.$; ${ }^{\star} p<0.01 ;{ }^{* \star} p<0.001$ ) apply to the differences in annual antibiotic purchase rates between progressors and non-progressors.

common in patients with type 1 diabetes compared with age-matched and sex-matched NDC subjects, and that the incidence of bacterial infections increased in parallel with the severity of diabetic nephropathy. The use of antibiotics also correlated with the long-term glycemic control in patients with normoalbuminuria, microalbuminuria, and macroalbuminuria. Frequent bacterial infections and recurrent use of antibiotics were also associated with an increased risk of incident microalbuminuria in patients with diabetes.

Previous studies have shown that patients with diabetes are at higher risk of hospitalization due to bacterial infections. ${ }^{13} 22$ On the basis of our hospital discharge register data, we observed that the risk of hospitalization due to bacterial infections was 4.3-fold higher in patients with type 1 diabetes, compared with NDCs. Our results also indicate that type 1 diabetes was associated with an increased risk of less severe infections, treated outside of hospitals. The overall number of annual antibiotic purchases was, on average, twofold higher in patients with diabetes than in control subjects. This difference might partly be explained by the general awareness of risks of infections related to diabetes itself, and it is possible that the threshold for clinicians to prescribe antimicrobial medications is lower, and the antibiotics may be prescribed in a prophylactic manner for patients with diabetes.

Albuminuria and reduced GFR have previously been associated with an increased risk of infection-related mortality. ${ }^{17} 18$ We observed that diabetic nephropathy at all stages was a risk factor for bacterial infections. Microalbuminuria did not increase the rate of hospitalization due to bacterial infections, but macroalbuminuria doubled the rate compared with patients with normal AER. Similar findings were found in the corresponding results for outpatient antibiotic purchases, where microalbuminuria increased the number of annual antibiotic purchases by 1.2 times and macroalbuminuria by 1.3 times. ESRD has previously been shown to be a strong risk factor for infectious diseases, ${ }^{16}$ and in our study dialysis increased the rate of hospital-treated bacterial infections by 11 times and the number of annual antibiotic purchases by 2.4 times compared to patients with diabetes and normal AER. Since long duration of diabetes increases the risk of diabetic complications, an important question that arose was how the duration of diabetes influenced the incidence of bacterial infections in patients with diabetes. We found that each year of the duration of diabetes increased the number of antibiotic purchases by $2.6 \%$. However, when adjusting for the nephropathy status, the increase was 1.4\%. Patients with incident microalbuminuria purchased significantly more antibiotics at the time of microalbuminuria diagnosis, compared with 4 years before the progression. These observations could suggest that bacterial infections may be associated with the development of diabetic nephropathy.

Hyperglycemia has been shown to cause immunosuppression, and to increase the susceptibility to bacterial 
infections. $^{7-9}{ }^{23}$ Several studies have demonstrated that poor glycemic control is a considerable risk factor for infections in patients with diabetes. ${ }^{1} 1024$ Although hyperglycemia could be a promoting factor for bacterial infections, infections themselves may also cause hyperglycemia. ${ }^{25}$ Hence, a vicious cycle may arise as frequent infections can worsen the glycemic control in patients with diabetes, and conversely, chronic hyperglycemia may facilitate the development of infections. ${ }^{26}$ We observed that poor glycemic control correlated with the number of antibiotic purchases, and that the relationship did not differ between the various nephropathy groups. The number of antibiotic purchases increased by $6-10 \%$ for each unit increase in HbAlc at all stages of nephropathy. As chronic hyperglycemia remains the primary cause of metabolic, biochemical, and vascular abnormalities in diabetic nephropathy, it could be argued that poor glycemic control may be the link between the progression of diabetic nephropathy and the higher incidence of bacterial infections.

Some limitations must be considered in our study. Data from the National Drug Prescription Register do not reveal the type or site of the infection for which the antibiotic treatment was required, only the compound itself, and the number of packages sold are recorded in the register. Based on the reports from the Finnish Medicines Agency, ${ }^{27}$ hospitals annually purchase about 15-20\% of all antimicrobial drug purchases in Finland, and these purchases are not recorded in the National Drug Prescription Register. Of note, most of these infection events are recorded in The Hospital Discharge Register, and included in our study as infections that led to hospitalizations. The Hospital Discharge Register also has its own drawbacks. Owing to limitations of the ICD-coding system, it can sometimes be challenging to retrospectively establish the specific etiology of the infections-whether they are viral or bacterial in origin. It should be noted that certain types of infections are much more often encountered in patients with diabetes than in patients without diabetes; for example, patients with diabetic neuropathy are prone to foot ulcers, which when infected can lead to osteomyelitis and possibly even bacteremia. Also, physicians often have a lower threshold for admitting patients with diabetes into hospital care due to an infection, compared to patients with an infection without any underlying disorders. This contributes to the large difference in the number of hospitalizations seen in patients with diabetes when compared with the NDC subjects.

Our study shows that bacterial infections are more frequent in patients with type 1 diabetes compared with age-matched and sex-matched NDCs, both in hospital and outpatient settings. Although our study cannot ascertain whether bacterial infections lead to the development and progression of diabetic nephropathy, or if the nephropathy predisposes the patient to bacterial infections, it does show that there is a strong association between the two. Bacterial infections could cause kidney injury via endotoxins, ${ }^{28} 29$ or by direct invasion of pathogens, which may lead to inflammation and renal scarring. Chronic hyperglycemia could also predispose to infections as well as the progression of nephropathy. More studies are required to elucidate which risk factors increase the susceptibility to bacterial infections in patients with type 1 diabetes, and to further investigate the association between bacterial infections and the risk of the development and progression of diabetic nephropathy.

\section{Author affiliations}

${ }^{1}$ Folkhälsan Institute of Genetics, Folkhälsan Research Center, Biomedicum Helsinki, Helsinki, Finland

${ }^{2}$ Division of Nephrology, Department of Medicine, Helsinki University Central Hospital, Helsinki, Finland

${ }^{3}$ Research Program Unit, Department of Diabetes and Obesity, University of Helsinki, Helsinki, Finland

${ }^{4}$ Diabetes Prevention Unit, National Institute for Health and Welfare, Helsinki, Finland

${ }^{5}$ Division of Infectious Diseases, Department of Medicine, Helsinki University Central Hospital, Helsinki, Finland

${ }^{6}$ Helsinki University Hospital Laboratory, Department of Bacteriology, Helsinki University Central Hospital, Helsinki, Finland

${ }^{7}$ Baker IDI Heart and Diabetes Institute, Melbourne, Victoria, Australia

Acknowledgements The authors thank the physicians and nurses at each FinnDiane Study center for their contribution to this work. See online supplementary file for members in the FinnDiane Study.

Contributors JRS and VH were responsible for data assembly and writing the manuscript. VH was responsible for statistical analyses. ML and AJ contributed to the conception and design of the study and critically revised the manuscript. CF was responsible for validation and interpretation of the phenotypic data as well as for critical revision of the article. JK and P-HG participated in the interpretation of the results and critical revision of the article. P-HG is also the guarantor for the study. All authors approved the final version of the manuscript.

Funding Folkhälsan Research Foundation (P-HG), Wilhelm and Else Stockmann Foundation (JRS, P-HG, ML), Liv och Hälsa Foundation (P-HG), Päivikki and Sakari Sohlberg Foundation (P-HG), Academy of Finland (134379 to $\mathrm{P}-\mathrm{HG}$ ), Diabetes Research Foundation (ML), and the Novo Nordisk Foundation (ML).

Competing interests P-HG has received lecture honoraria from Abbot, Boehringer Ingelheim, Cebix, Eli Lilly, Genzyme, Novartis, Novo Nordisk, and MSD, and research grants from Eli Lilly and Roche. He is also an advisory board member for Boehringer Ingelheim, Novartis, and Medscape.

\section{Patient consent Obtained.}

Ethics approval Ethics Committee, Department of Medicine, Helsinki, Finland. Provenance and peer review Not commissioned; externally peer reviewed.

\section{Data sharing statement No additional data are available.}

Open Access This is an Open Access article distributed in accordance with the Creative Commons Attribution Non Commercial (CC BY-NC 4.0) license, which permits others to distribute, remix, adapt, build upon this work noncommercially, and license their derivative works on different terms, provided the original work is properly cited and the use is non-commercial. See: http:// creativecommons.org/licenses/by-nc/4.0/

\section{REFERENCES}

1. Shah BR, Hux JE. Quantifying the risk of infectious diseases for people with diabetes. Diabetes Care 2003;26:510-13. 
2. Bertoni AG, Saydah S, Brancati FL. Diabetes and the risk of infection-related mortality in the U.S. Diabetes Care 2001;24:1044-9.

3. Seshasai SR, Kaptoge S, Thompson A, et al., The Emerging Risk Factor Collaboration. Diabetes mellitus, fasting glucose, and risk of cause-specific death. N Engl J Med 2011;364:829-41.

4. Thomsen RW, Hundborg $\mathrm{HH}$, Lervang $\mathrm{HH}$, et al. Risk of community-acquired pneumococcal bacteremia in patients with diabetes: a population-based case-control study. Diabetes Care 2004;27:1143-7.

5. Thomsen RW, Hundborg $\mathrm{HH}$, Lervang $\mathrm{HH}$, et al. Diabetes mellitus as a risk and prognostic factor for community-acquired bacteremia due to enterobacteremia: a 10-year population based study among adults. Clin Infect Dis 2005;40:628-31.

6. Thomsen RW, Riis AH, Kjeldsen S, et al. Impact of diabetes and poor glycaemic control on risk of bacteremia with haemolytic streptococci groups A, B, and G. J Infect 2011;63:8-16.

7. Valerius $\mathrm{NH}, \mathrm{Eff} \mathrm{C}$, Hansen $\mathrm{NE}$, et al. Neutrophil and lymphocyte function in patients with diabetes mellitus. Acta Med Scand 1982;211:463-7.

8. Marhoffer W, Stein M, Maeser E, et al. Impairment of polymorphonuclear leukocyte function and metabolic control of diabetes. Diabetes Care 1992;15:256-60.

9. Delamaire $M$, Maugendre D, Moreno M, et al. Impaired leucocyte functions in diabetic patients. Diabet Med 1997;14:29-34.

10. Muller LMAJ, Gorter KJ, Hak E, et al. Increased risk of common infections in patients with type 1 and type 2 diabetes mellitus. Clin Infect Dis 2005;41:281-8.

11. Gordin D, Forsblom C, Rönnback M, et al. Acute hyperglycaemia induces an inflammatory response in young patients with type 1 diabetes. Ann Med 2008;40:627-33.

12. Cherney DZ, Scholey JW, Sochett E, et al. The acute effect of clamped hyperglycemia on the urinary excretion of inflammatory cytokines/chemokines in uncomplicated type 1 diabetes: a pilot study. Diabetes Care 2011;34:177-80.

13. Benfield T, Jensen JS, Nordestgaard BG. Influence of diabetes and hyperglycaemia on infectious disease hospitalization and outcome. Diabetologia 2007;50:549-54.

14. Vinik Al, Maser RE, Mitchell BD, et al. Diabetic autonomic neuropathy. Diabetes Care 2003;26:1553-79.
15. Kim PJ, Steinberg JS. Complications of the diabetic foot. Endocrinol Metab Clin North Am 2013;42:833-47.

16. Choncol M. Neutrophil dysfunction and infection risk in end-stage renal disease. Semin Dial 2006;19:291-6.

17. Wang HE, Gamboa C, Warnock DG, et al. Chronic kidney disease and risk of death from infection. Am J Nephrol 2011;34:330-6.

18. James MT, Laupland KB, Tonelli M, et al. Risk of bloodstream infection in patients with chronic kidney disease not treated with dialysis. Arch Intern Med 2008;168:2333-9.

19. Saraheimo M, Teppo AM, Forsblom C, et al. Diabetic nephropathy is associated with low-grade inflammation in type 1 diabetic patients. Diabetologia 2003;46:1402-7.

20. Fornoni A, ljaz A, Tejada T, et al. Role of inflammation in diabetic nephropathy. Curr Diabetes Rev 2008;4:10-17. .

21. Min Y, Agresti A. Random effect models for repeated measures of zero-inflated count data. Stat Model 2005;5:1-19.

22. Hamilton EJ, Martin N, Makepeace A, et al. Incidence and predictors of hospitalization for bacterial infection in community-based patients with type 2 diabetes: the Fremantle diabetes study. PLOS ONE 2013;8:e60502.

23. Kawahito S, Kitahata H, Oshita S. Problems associated with glucose toxicity: role of hyperglycemia-induced oxidative stress. World $\mathrm{J}$ Gastroenterol 2009; 15:4137-42.

24. Kornum JB, Thomsen RW, Riis A, et al. Diabetes, glycemic control, and risk of hospitalization with pneumonia: a population based case-control study. Diabetes Care 2008;31:1541-5.

25. Kitabchi AE, Umpierrez GE, Murphy MB, et al. Management of hyperglycemic crises in patients with diabetes. Diabetes Care 2001;24:131-53.

26. Boyanova L, Mitov I. Antibiotic rates in causative agents of infections in diabetic patients: rising concerns. Expert Rev Anti Infect Ther 2013;11:411-20.

27. Fimea; Lääkekulutus vuosina 2009-2012. http://raportit.nam.fi/ raportit/kulutus/laakekulutus.htm (accessed 12 Apr 2014).

28. Doi K, Leelahavanichkul A, Yuen PS, et al. Animal models of sepsis and sepsis-induced kidney injury. J Clin Invest 2009;119:2868-78.

29. Nymark M, Pussinen PJ, Tuomainen AM, et al. Serum lipopolysaccharide activity is associated with the progression of kidney disease in Finnish patients with type 1 diabetes. Diabetes Care 2009;32:1689-93. 